\title{
Sulfatase 1 mediates the inhibitory effect of angiotensin II type 2 receptor inhibitor on angiotensin II-induced hypertensive mediator expression and proliferation in vascular smooth muscle cells from spontaneously hypertensive rats
}

\author{
Hye Young Kim, Hye Ju Cha, Hee Sun Kim \\ Department of Microbiology, Yeungnam University College of Medicine, Daegu, Korea
}

Background: Extracellular sulfatases (Sulfs), sulfatase 1 (Sulf1) and sulfatase 2 (Sulf2), play a pivotal role in cell signaling by remodeling the 6-O-sulfation of heparan sulfate proteoglycans on the cell surface. The present study examined the effects of Sulfs on angiotensin II (Ang II)-induced hypertensive mediator expression and vascular smooth muscle cells (VSMCs) proliferation in spontaneously hypertensive rats (SHR). Methods: Ang II receptors, 12-lipoxygenase (12-LO), and endothelin-1 (ET-1) messenger RNA (mRNA) expressions in SHR VSMCs were analyzed by real-time polymerase chain reaction and Western blotting. VSMCs proliferation was determined by $\left[{ }^{3} \mathrm{H}\right]$-thymidine incorporation.

Results: Basal Sulfs mRNAs expression and enzyme activity were elevated in SHR VSMCs. However, Sulfs had no effect on the basal or Ang II-induced 12-LO and ET-1 mRNA expression in SHR VSMCs. The inhibition of Ang II-induced 12-LO and ET-1 expression by blockade of the Ang II type 2 receptor (AT 2 R) pathway was not observed in Sulf1 siRNA-transfected SHR VSMCs. However, Sulf2 did not affect the action of $A T_{2} R$ inhibitor on Ang II-induced 12-LO and ET-1 expression in SHR VSMCs. The down-regulation of Sulf1 induced a reduction of $A T_{2} R$ mRNA expression in SHR VSMCs. In addition, the inhibition of Ang II-induced VSMCs proliferation by blockade of the $A T_{2} R$ pathway was mediated by Sulf1 in SHR VSMCs. Conclusion: These findings suggest that extracellular sulfatase Sulf1 plays a modulatory role in the $A T_{2}$ $\mathrm{R}$ pathway that leads to an Ang II-induced hypertensive effects in SHR VSMCs.

Keywords: Sulfatases; Angiotensin II type 2 receptor; Hypertension

\section{INTRODUCTION}

Extracellular sulfatases (Sulfs) are hydrolytic enzymes that regulate cell metabolism and signaling [1]. There are 17 distinct sulfatases in humans and 14 sulfatases in rodents. Sulfa-

Received: April 26, 2017, Revised: May 15, 2017

Accepted: May 22, 2017

Corresponding Author: Hee Sun Kim, Department of Microbiology, Yeungnam University College of Medicine,

170 Hyeonchung-ro, Nam-gu, Daegu 42415, Korea

Tel: +82-53-640-6942, Fax: +82-53-653-6628

E-mail: heesun@med.yu.ac.kr tases are classified as lysosomal, nonlysosomal, and Sulfs, according to their localization [1,2]. Sulfs are designated as heparin sulfate 6-O-endosulfatases, and to date, human, mouse, and rat orthologs have been cloned [3,4]. There are two kinds of heparin sulfate 6-O-endosulfatases: sulfatase 1 (Sulf1) or sulfatase FP1 (SulfFP1) and sulfatase 2 (Sulf2) or sulfatase FP2 (SulfFP2) [4]. Sulf1 and Sulf2 regulate cell signaling by remodeling the heparan sulfate proteoglycans (HSPGs) on the surface of cells. HSPGs bind to growth factors, inhibiting their downstream signaling pathways. Removal of 6-O-sulfate groups from HSPGs via sulfatases leads to the release of bound growth factors, which modulate signaling pathways [1,3].

Copyright ( 12017 Yeungnam University College of Medicine

This is an Open Access article distributed under the terms of the Creative Commons Attribution Non-Commercial License (http://creativecommons.org/licenses/by-nc/4.0/) which permits unrestricted non-commercial use, distribution, and reproduction in any medium, provided the original work is properly cited. 
Therefore, altered Sulfs activity plays an important role in both cell survival and proliferation. In addition to cell signaling, Sulfs modulate various cellular processes, such as cell development, tumor growth, muscle regeneration, neuromodulation, and immunomodulation [1,5-7]. Mice with double knockout Sulf1 and Sulf2 show significant developmental defects and reduced body weight [8,9]. Although Sulf1 and Sulf2 are structurally similar and modulate cell signaling, they have opposite effects in tumor cells. Sulf1 in cancer cells is known to inhibit angiogenesis and proliferation [10,11]. Contrastingly, Sulf2 has pro-angiogenic and tumorigenic effects $[12,13]$. Therefore, Sulfs have been proposed to be novel reagents for modifying HSPGs or therapeutic targets for cancer treatment.

The pathophysiological roles of Sulfs in the development or maintenance of hypertension have not yet been fully elucidated. HSPGs are found in vascular walls, and reduced HSPG levels in the glomerular basement membrane are associated with essential hypertension [14]. Sulfs are known to regulate 6-O-sulfation of HSPGs; thus, they have been associated with the mediation of several functions of vascular smooth muscle cells (VSMCs) $[13,15]$. Sulfs may play a functional role in the development and maintenance of hypertensive vasculature since expression of hypertensive mediators and proliferation of VSMCs are important pathologic features of hypertension.

To the best of our knowledge, there have been no studies evaluatibg the relationship between Sulfs and the development and maintenance of hypertension. Although Sulfs have been established to mediate cell signaling and proliferation, the exact mechanism of Sulfs in hypertensive VSMCs have not been fully elucidated. Therefore, as a preliminary study to determine the relationship between Sulfs activity and pathophysiologic process of hypertensive cells, we examined the effects of Sulfs on angiotensin II (Ang II)-induced hypertensive mediators, 12-lipoxygenase (12-LO), and endothelin-1 (ET-1) expression, as well as cell proliferation in VSMCs from spontaneously hypertensive rats (SHR).

\section{MATERIALS AND METHODS}

\section{Reagents}

Total RNA extraction kit was purchased from iNtRON
(Biotechnology Inc, Seoul, Korea). Dulbecco’s Modified Eagle's medium (DMEM), fetal bovine serum (FBS) and penicillinstreptomycin were purchased from Lonza (Walkersville, MD, USA). Ang II was purchased from Calbiochem (San Diego, CA, USA). Losartan and PD123319 were purchased from Sigma-Aldrich Co. (St. Louis, MO, USA). LightCycler FastStart DNA SYBR Green I Mix was purchased from Roche (Mannheim, Germany). Lipofectamine 2000 was purchased from Invitrogen (Carlsbad, CA, USA). Primer oligomers for Ang II type 1 receptor $\left(\mathrm{AT}_{1} \mathrm{R}\right)$, Ang II type 2 receptor $\left(\mathrm{AT}_{2} \mathrm{R}\right)$, Sulf1, Sulf2, 12-LO, ET-1, and $\beta$-actin were synthesized at Bionics (Daejeon, Korea). 12-LO and ET-1 antibodies were purchased from Santa Cruz Biotechnology (California, USA). $\mathrm{AT}_{2} \mathrm{R}$ antibody was purchased from Abcam (Cambridge, UK). Monoclonal anti- $\gamma$-tubulin antibody was purchased from SigmaAldrich (St. Louis, MO, USA). Rat Sulf1 small interfering RNA (siRNA) oligomers were purchased from Bioneer technology (Daejeon, Korea). Rat Sulf2 siRNA oligomers were purchased from Santa Cruz Biotechnology (California, USA). Negative control siRNA was purchased from Invitrogen (Carlsbad, CA, USA). All other reagents used in this study were pure-grade commercial preparations.

\section{Animals}

Specific pathogen-free, inbred normotensive, male WistarKyoto rats (WKY) and SHR, all aged 22-weeks, were purchased from Japan SLC Inc. (Shizuoka, Japan). All experimental animals were fed autoclaved food and received bedding in order to minimize exposure to microbial pathogens. This experiment was approved by the Institutional Animal Care and Use Committee of the Yeungnam University College of Medicine (protocol no. YUMC-AEC2013-006).

\section{Preparation of VSMCs}

VSMCs were isolated from the thoracic aortas of these rats, following the explant method [16]. VSMCs were cultured in DMEM, which had been supplemented with 10\% FBS and 1\% penicillin-streptomycin. The cells were detached with $0.25 \%$ trypsin/EDTA and seeded into $75 \mathrm{~cm}^{2}$ tissue culture flasks at a density of 105 cells $/ \mathrm{mL}$. All experiments were conducted during cell passages 3 to 7. Prior to stimulation, 95\% confluent VSMCs were serum-starved overnight in 
DMEM containing $0.1 \%$ FBS. The cell cultures were incubated in a humidified incubator at $37^{\circ} \mathrm{C}$ and $5 \% \mathrm{CO}_{2}$ in the presence or absence of stimuli for the indicated time.

\section{Preparation of total RNA and real-time poly- merase chain reaction (PCR)}

Total RNA was isolated using an easy-BLUE total RNA extraction kit (iNtRON Biotechnology, Seoul, Korea). $\mathrm{AT}_{1}$ $\mathrm{R}, \mathrm{AT}_{2} \mathrm{R}$, Sulf1, Sulf2, 12-LO, and ET-1 complementary DNAs (cDNAs) were obtained from VSMCs by reverse transcription of $1 \mu \mathrm{g}$ of total RNA. Reverse transcription of the RNA was performed using oligo dT primer Maxime RT PreMix (iNtRON Biotechnology, Seoul, Korea). cDNAs were then subjected to real-time PCR using a LightCycler with the FastStart DNA Master SYBR Green kit. PCR amplification was performed as previously described by Kim et al. [16]. The primers used for PCR were as follows: $\mathrm{AT}_{1} \mathrm{R}(445 \mathrm{bp})$ sense, 5'-cacctatgtaagatcgcttc-3', antisense, 5'-gcacaatcgccataattatcc-3'; $\mathrm{AT}_{2} \mathrm{R}$ (65 bp) sense, 5'-ccgtgaccaagtcttgaagatg-3', antisense, 5'-agggaagccagcaaatgatg-3'; Sulf1(136 bp) sense, 5'-aaacagtgcaacccaagacc-3', antisense, 5'-ttgccagttggtgtctgaag3'; Sulf2 (73 bp) sense, 5'-ggcttagagacggaggaag-3', antisense, 5'-ggtctcttcatttctggcca-3'; 12-LO (312 bp) sense, 5'-tggggcaactggaagg-3', antisense, 5'-agagcgcttcagcaccat-3'; ET-1 (370 bp) sense, 5 '-ctcctccttgatggacaagg-3', antisense, 5 '-cttgatgctgttgctcatgg-3'; and $\beta$-actin (101 bp) sense, 5'-tactgccetggctcctagca3 ', antisense, 5'-tggacagtgaggccaggatag-3'. mRNA level of each sample was normalized to the mRNA level of $\beta$-actin, a housekeeping gene.

\section{Measurement of sulfatase activity}

The activity levels of Sulfs in cell lysate $(30 \mu \mathrm{g})$ were measured using a sulfatase activity assay kit obtained from Abcam (Cambridge, UK). All procedures were performed in accordance with the manufacturer's instructions.

\section{Western blotting}

Total lysates were prepared in PRO-PREP buffer (iNtRON, South Korea). Protein concentrations were determined by the Bradford assay (Bio-Rad, Richmond, CA, USA), using bovine serum albumin as the standard. Twenty-microgram protein samples were separated on 10\% SDS-polyacrylamide gels, and then transferred to the nitrocellulose membranes. The membranes were soaked in 5\% nonfat dried milk in tris buffered saline (TBST, $10 \mathrm{mmol} / \mathrm{L}$ Tris-HCl pH 7.5, $150 \mathrm{mmol} / \mathrm{L}$ $\mathrm{NaCl}$ and $0.05 \%$ Tween-20) for $1 \mathrm{~h}$, and then incubated for 16-18 $\mathrm{h}$ with polyclonal antibody against $\mathrm{AT}_{2} \mathrm{R}$ (dilution 1/800), 12- LO (dilution 1/400), ET-1 (dilution 1/400), and monoclonal antibody against $\gamma$-tubulin (dilution $1 / 2,000$ ) at $4^{\circ} \mathrm{C}$. The membranes were washed three times with TBST for $10 \mathrm{~min}$, and then incubated with horseradish peroxidaseconjugated secondary antibody for $1 \mathrm{~h}$ at $4{ }^{\circ} \mathrm{C}$. The membranes were rinsed three times with TBST for $10 \mathrm{~min}$, and the antigen-antibody complex was detected using the enhanced chemiluminescence detection system (LAS-3000, Fujifilm, Japan).

\section{Small interfering RNA}

VSMCs were plated on 6-well plates and grown to 90\% confluence. VSMCs were then transfected with Sulf1 or Sulf2 siRNA oligomers $(50 \mathrm{nmol} / \mathrm{L})$, using lipofectamine 2000 in accordance with the manufacturer's instructions. After $24 \mathrm{~h}$ of incubation, VSMCs were placed a growth medium for $24 \mathrm{~h}$ before starting the experiments. The cells were then cultured in the presence or absence of stimuli for $2 \mathrm{~h}$. The sense and antisense oligonucleotides were as follows: Sulf1 siRNA sense, 5'-gugacuucaggaaugagau-3', antisense, 5'-aucucauuccugaagucac-3'; and Sulf2 siRNA sense, 5'-cacaucacaccgaguuaca-3', antisense, 5'-uguaacucggugugaugug- 3 '.

\section{VSMCs proliferation}

VSMCs were plated on 24-well plates with serum-free medium for $24 \mathrm{~h}$ and then exposed to stimulant. $\left[{ }^{3} \mathrm{H}\right]$-thymidine $(1 \mu \mathrm{Ci} / \mathrm{mL})$ (Perkin Elmer precisely, Boston, MA, USA) was added to the plates during the last $24 \mathrm{~h}$ of incubation. The cells were subsequently washed three times with cold PBS. $\left[{ }^{3} \mathrm{H}\right]$-thymidine-labeled cells were collected with $0.1 \%$ SDS, and radioactivity was counted using a Packard scintillation counter (Packard Instrument Company, Meriden, CT, USA).

\section{Statistical analysis}

The results were expressed as the means \pm SEM of at least three or four independent experiments. Statistical significance 
was determined by Student's t-test. A p-value of less than 0.05 was considered as statistically significant. Statistical analysis was performed using IBM SPSS version 23.0 (IBM Co., Armonk, NY, USA).

\section{RESULTS}

\section{Gene expression and enzyme activity of Sulfs are elevated in SHR VSMCs}

The basal expression levels of Sulfs mRNAs and enzyme activity were examined in WKY and SHR VSMCs. The basal expression levels of Sulf1 and Sulf2 mRNAs in SHR VSMCs were compared with those in WKY VSMCs (Fig. 1A). The enzyme activity of Sulfs in SHR VSMCs was also higher than that in WKY VSMCs (Fig. 1A). In addition, we observed the direct effect of Ang II on Sulf1 and Sulf2 mRNA expressions in bothe WKY and SHR VSMCs. Ang II reduced the expression levels of Sulf1 and Sulf2 mRNA, as well as Sulfs activity in both WKY and SHR VSMCs (Fig. 1A). Next, we examined the effects of Sulf1 and Sulf2 on mRNA expressions of Ang
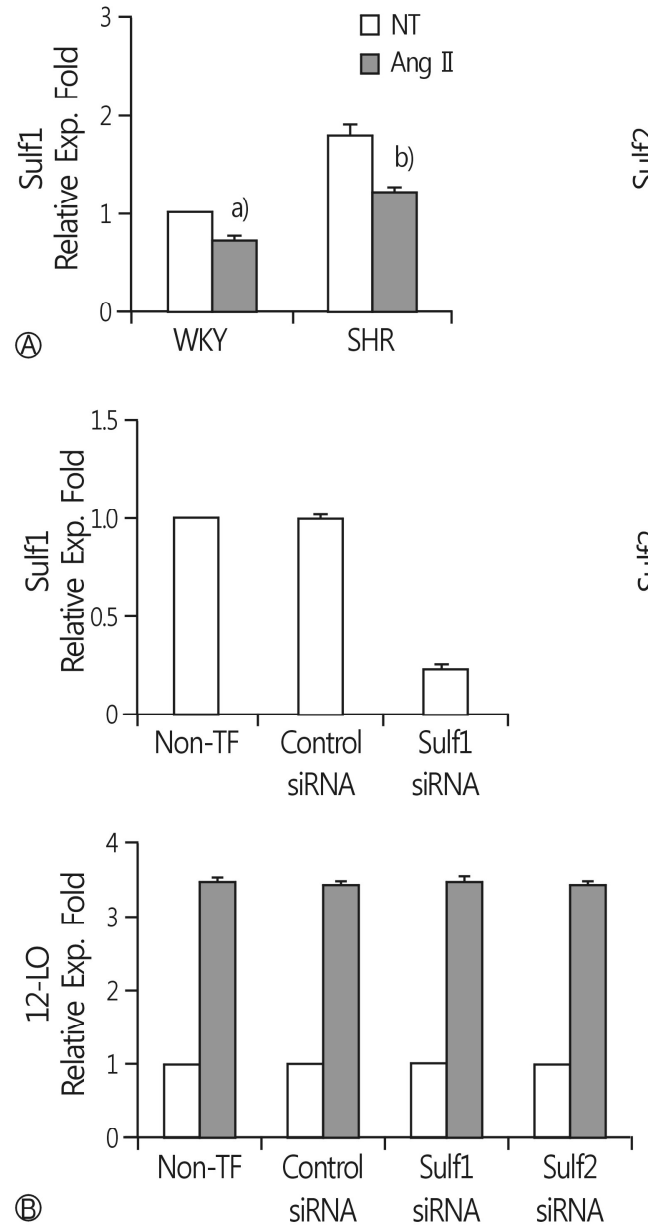
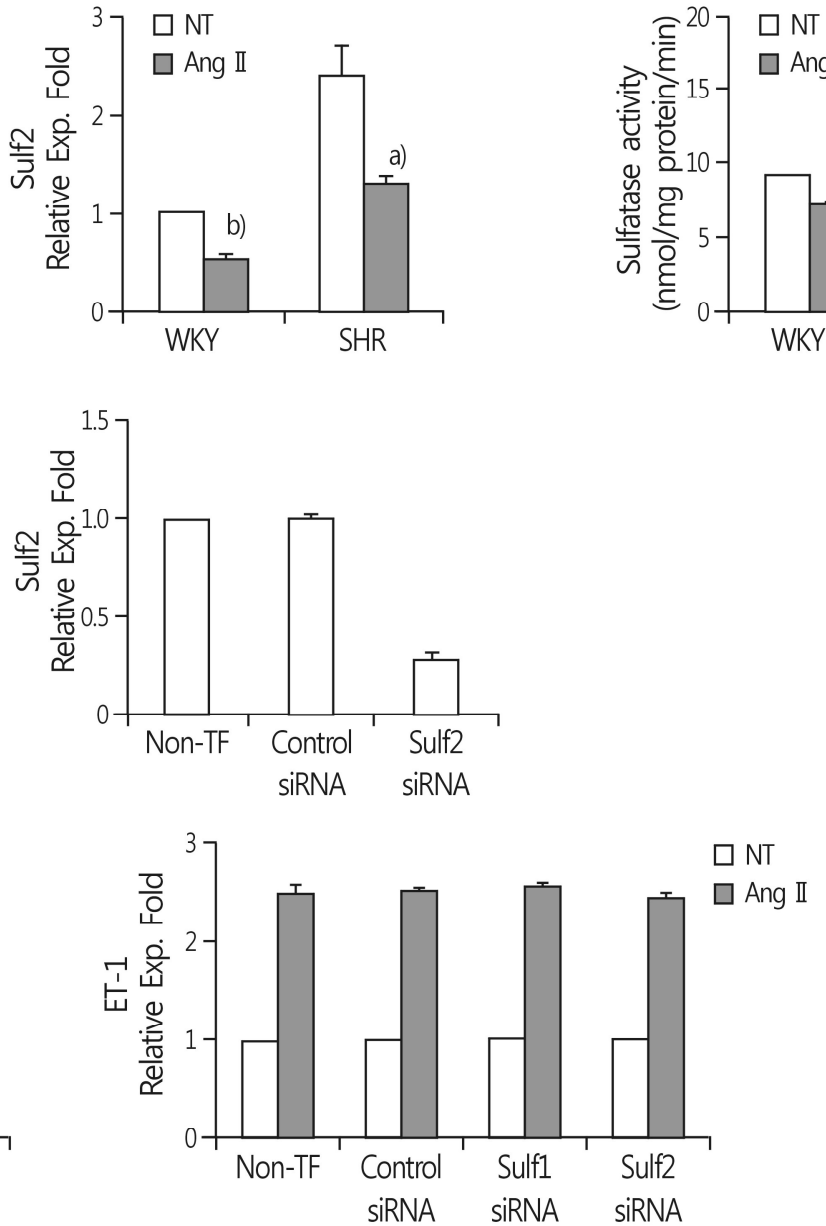

Fig. 1. Gene expression and activity of Sulfs are increased in SHR VSMCs, and Sulfs have no effect on expression levels of Ang II-induced 12-LO and ET-1 mRNA in SHR VSMCs. (A) WKY and SHR VSMCs were untreated (NT) or treated with Ang II (0.1 $\mu \mathrm{mol} / \mathrm{L})$ for $2 \mathrm{~h}$. After total RNAs or cell lysates were isolated, real-time PCR or sulfatase activity assay was performed. Bars represent means \pm SEM from three independent experiments. ${ }^{a} p<0.05,{ }^{b} p<0.01,{ }^{c} p<0.001$ vs. untreated WKY or SHR VSMCs. (B) SHR VSMCs were plated on 6-well plates, grown to $90 \%$ confluence, and transfected with Sulf1, Sulf2, or control siRNA oligomers (50 nmol/L). Total RNAs were analyzed by real-time PCR to confirm successful transfection. Additionally transfected VSMCs were untreated (NT) or treated with Ang II $(0.1 \mu \mathrm{mol} / \mathrm{L})$ for $2 \mathrm{~h}$, after which 12-LO or ET-1 mRNA expression was determined by real-time PCR. Non-TF: non-transfected SHR VSMCs. Bars represent the means \pm SEM of three independent experiments. Sulfs, extracellular sulfatases; Ang II, angiotensin II; WKY, Wistar-Kyoto rats; SHR, spontaneously hypertensive rats; 12-LO, 12-lipoxigenase; ET-1, endothelin-1; VSMCs, vascular smooth muscle cells; Sulf1, sulfatase 1; Sulf2, sulfatase 2; siRNA, small interfering RNA. 
II-induced hypertensive mediators, 12-LO and ET-1, in SHR VSMCs. Real-time PCR was performed after Sulf1 or Sulf2directed siRNA was transfected into SHR VSMCs. Both Sulf1 and Sulf2 had no statistically significant effect on the basal or Ang II-induced 12-LO and ET-1 mRNA expressions in SHR VSMCs (Fig. 1B).

\section{Sulf1 mediates inhibition of Ang Il-induced 12-LO and ET-1 mRNA expressions by $\mathrm{AT}_{2}$ $R$ inhibitor in SHR VSMCs}

Expressions of Ang II-induced 12-LO and ET-1 mRNA are mediated through the $\mathrm{AT}_{1} \mathrm{R}$ and $\mathrm{AT}_{2} \mathrm{R}$ pathways $[17,18]$.
Although Sulf1 and Sulf2 did not affect the expressions of Ang II-induced 12-LO and ET-1 mRNA, the activity of Sulfs was elevated in SHR VSMCs, while Ang II inhibited the gene expression and activity of Sulfs. Therefore, we examined the effects of Sulf1 and Sulf 2 on the $\mathrm{AT}_{1} \mathrm{R}$ or $\mathrm{AT}_{2} \mathrm{R}$ pathways, that mediate, Ang II-induced 12-LO and ET-1 mRNA expressions in SHR VSMCs. Down-regulation of Sulf1 did not affect the inhibition of Ang II-induced 12-LO and ET-1 mRNA expressions by the $\mathrm{AT}_{1} \mathrm{R}$ inhibitor losartan, but did affect the inhibition of Ang II-induced 12-LO and ET-1 mRNA expressions by the $\mathrm{AT}_{2} \mathrm{R}$ inhibitor, PD123319 (Fig. 2A). The inhibition of Ang II-induced 12-LO and ET-1 mRNA expression by $\mathrm{AT}_{2} \mathrm{R}$ inhibitor was not detected in

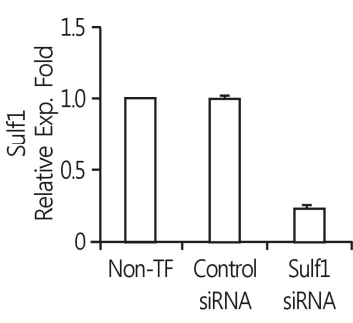

(A)

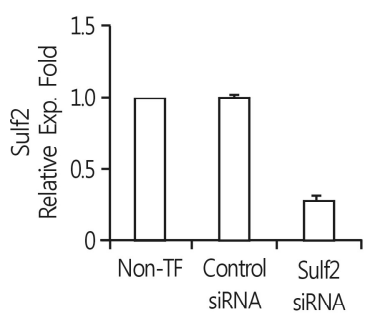

(B)
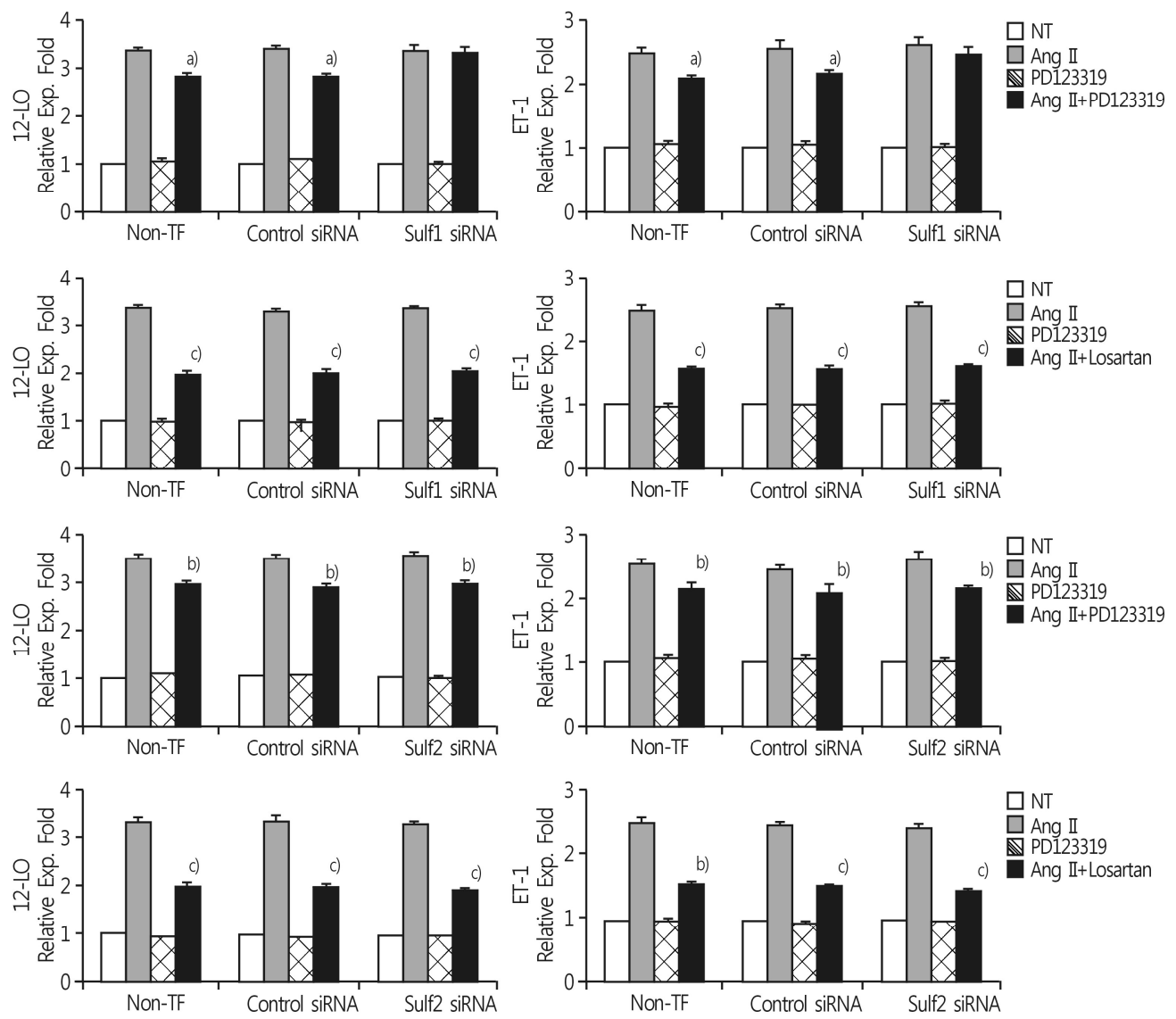

Fig. 2. Expression of Ang II-induced 12-LO and ET-1 mRNA is not inhibited by AT 2 R inhibitor, PD123319, in Sulf1 siRNA-transfected SHR VSMCs. SHR VSMCs were plated on 6-well plates, grown to 90\% confluence, and transfected with Sulf1 (A), Sulf2 (B), or control siRNA oligomers $(50 \mathrm{nmol} / \mathrm{L})$. Total RNAs were analyzed by real-time PCR to confirm successful transfection. Additionally, SHR VSMCs were treated with or without Ang II $(0.1 \mu \mathrm{mol} / \mathrm{L})$ in the presence or absence of losartan $\left(\mathrm{AT}_{1} \mathrm{R}\right.$ inhibitor, $\left.10 \mu \mathrm{mol} / \mathrm{L}\right)$ or PD123319 (AT 2 R inhibitor, $10 \mu \mathrm{mol} / \mathrm{L}$ ) for $2 \mathrm{~h}$, after which total RNAs were analyzed by real-time PCR. Bars represent the means \pm SEM of three inde- pendent experiments. ${ }^{\mathrm{a}} p<0.05,{ }^{\mathrm{b})} p<0.01,{ }^{\mathrm{c})} p<0.001$ vs. non-transfected or transfected SHR VSMCs treated with Ang II. Ang II, angiotensin II; 12-LO, 12-lipoxigenase; ET-1, endothelin-1; $\mathrm{AT}_{2} \mathrm{R}$, angiotensin II type 2 receptor; VSMCs, vascular smooth muscle cells; SHR, spontaneously hypertensive rats; Sulf1, sulfatase 1; Sulf2, sulfatase 2. 
Sulf1 siRNA-transfected SHR VSMCs. However, the downregulation of Sulf2 did not affect the inhibition of Ang IIinduced 12-LO and ET-1 mRNA expression by losartan or PD123319 in SHR VSMCs (Fig. 2B). To confirm the effect of Sulf1 on the $\mathrm{AT}_{2} \mathrm{R}$ pathway, we performed a Western blot analysis to observe the inhibition of Ang II-induced 12$\mathrm{LO}$ and ET-1 protein production by $\mathrm{AT}_{2} \mathrm{R}$ inhibitor in SHR VSMCs transfected with Sulf1 siRNA. Down-regulation of Sulf1 reduced the inhibition of Ang II-induced 12-LO and ET-1 protein production by PD123319 (Fig. 3). The rate of inhibition of Ang II-induced 12-LO protein production by PD123319 in Sulf1 siRNA-transfected SHR VSMCs was reduced to $3.8 \pm 0.4 \%$, when compared with $19.9 \pm 0.4 \%$ in the control siRNA-transfected SHR VSMCs. In the case of ET-1 protein expression, the rate of inhibition of Sulf1 siRNA-transfected SHR VSMCs was reduced to $6.0 \pm 2.5 \%$, when compared with $21.5 \pm 1.4 \%$ in the control siRNA-transfected SHR VSMCs.

We subsequently examined whether Sulf1 or Sulf2 has any mediating effect on the inhibition of Ang II-induced 12-LO and ET-1 mRNA expressions by $\mathrm{AT}_{1} \mathrm{R}$ or $\mathrm{AT}_{2} \mathrm{R}$ inhibitor in WKY VSMCs. Contrary to SHR VSMCs, down-regulation of Sulf1 did not alter the inhibitory effect of PD123319 on Ang II-induced 12-LO and ET-1 mRNA expressions (Fig. 4). Neither Sulf1 nor Sulf2 mediated the inhibitory effect of $\mathrm{AT}_{1}$ $\mathrm{R}$ or $\mathrm{AT}_{2} \mathrm{R}$ inhibitor on Ang II-induced 12-LO and ET-1
mRNA expressions in WKY VSMCs.

\section{Sulf1 maintains expression of $A T_{2} R$ in SHR VSMCs}

We examined the direct effects of Sulf1 and Sulf2 on the expression of Ang II-induced $\mathrm{AT}_{1} \mathrm{R}$ and $\mathrm{AT}_{2} \mathrm{R}$ mRNAs in SHR VSMCs. Real-time PCR was performed after Sulf1 or Sulf2-directed siRNA was transfected into SHR and WKY VSMCs. Sulf1 and Sulf2 had no statistically significant effect on the $\mathrm{AT}_{1} \mathrm{R}$ mRNA expression in both WKY and SHR VSMCs and no statistically significant effect on the $\mathrm{AT}_{2} \mathrm{R}$ mRNA expression in WKY VSMCs (Fig. 5A). Although the rate of reduction of the $\mathrm{AT}_{2} \mathrm{R}$ mRNA expression was low, a down-regulation of Sulf1 resulted in lowered $\mathrm{AT}_{2} \mathrm{R}$ mRNA expression in SHR VSMCs. Contrastingly, a down-regulation of Sulf2 had no effect on the $\mathrm{AT}_{2} \mathrm{R}$ mRNA expression in SHR VSMCs (Fig. 5A). We also observed a reduction of $\mathrm{AT}_{2}$ $\mathrm{R}$ protein production in Sulf1 siRNA-transfected SHR VSMCs (Fig. 5B).

\section{Inhibition of Ang II-induced VSMCs pro- liferation by $A T_{2} R$ inhibitor is mediated through Sulf1 in SHR VSMCs}

Sulfs have been established to mediate cell proliferation; Ang II have been known to induce VSMCs proliferation [17].
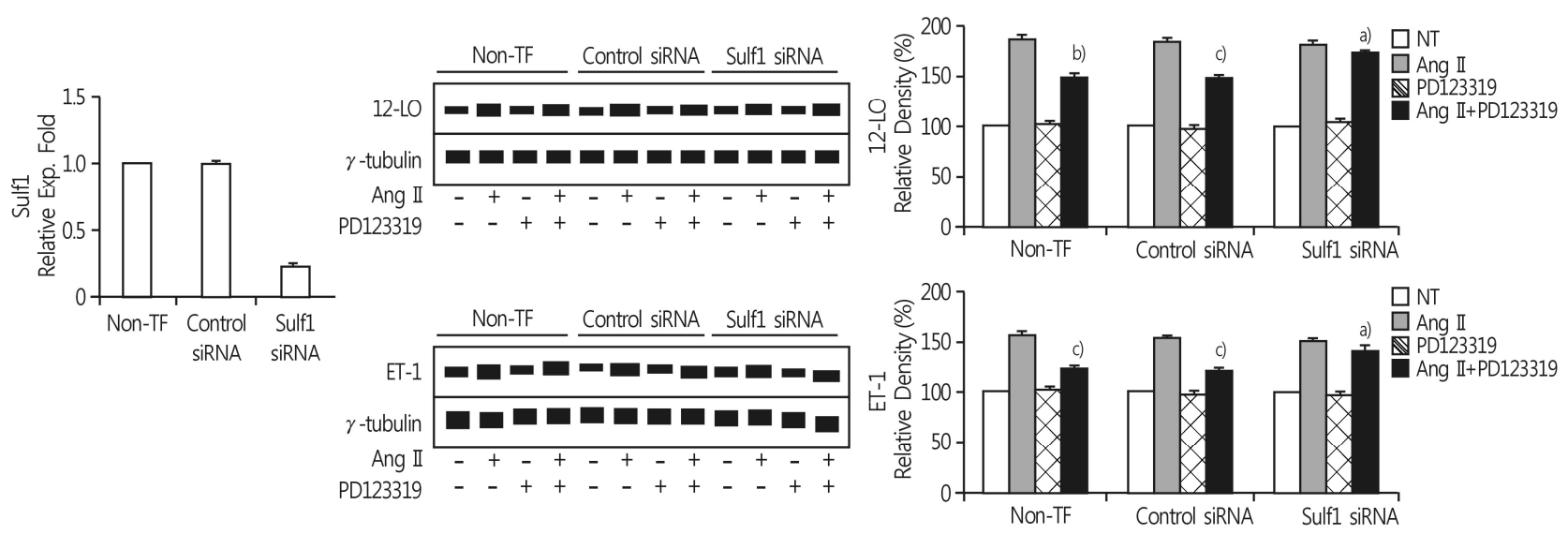

Fig. 3. Sulf1 mediates inhibition of Ang II-induced 12-LO and ET-1 expression by $\mathrm{AT}_{2} \mathrm{R}$ inhibitor in SHR VSMCs. SHR VSMCs were plated on 6-well plates, grown to $90 \%$ confluence, and transfected with Sulf1 or control siRNA oligomers (50 nmol/L). Total RNAs were analyzed by real-time PCR to confirm successful transfection. Additionally, SHR VSMCs were treated with or without Ang II $(0.1 \mu \mathrm{mol} / \mathrm{L})$ in the presence or absence of PD123319 $\left(\mathrm{AT}_{2} \mathrm{R}\right.$ inhibitor, $\left.10 \mu \mathrm{mol} / \mathrm{L}\right)$ for $2 \mathrm{~h}$, after which $12-\mathrm{LO}$ and ET-1 protein expression was determined by immunoblotting and densitometric analyses. Data shown are representative of three independent experiments. Bars represent the means \pm SEM of three independent experiments. ${ }^{\text {a) }} p<0.05,{ }^{\text {b) }} p<0.01,{ }^{c)} p<0.001$ vs. non-transfected or transfected SHR VSMCs treated with Ang II. Sulf1, sulfatase 1; Ang II, angiotensin II; 12-LO, 12-lipoxigenase; ET-1, endothelin-1; $\mathrm{AT}_{2} \mathrm{R}$, angiotensin II type 2 receptor; SHR, spontaneously hypertensive rats; VSMCs, vascular smooth muscle cells. 
Thus, we investigated whether Sulf1 or Sulf2 mediates Ang II-induced SHR VSMCs proliferation. Ang II-induced VSMCs proliferation is mediated through both the $\mathrm{AT}_{1} \mathrm{R}$ and $\mathrm{AT}_{2}$ $\mathrm{R}$ pathways. Therefore, we examined the effects of Sulf1 and Sulf2 on the inhibition of Ang II-induced VSMCs proliferation via the $\mathrm{AT}_{1} \mathrm{R}$ or $\mathrm{AT}_{2} \mathrm{R}$ inhibitor in SHR VSMCs. First, both Sulf1 and Sulf2 showed to have no effect on Ang II-induced VSMCs proliferation, as well as basal VSMCs proliferation (Fig. 6A). Second, Sulf1 or Sulf2 did not affect the inhibition of Ang II-induced VSMCs proliferation by the $\mathrm{AT}_{1}$ $\mathrm{R}$ inhibitor; losartan, and Sulf2 did not alter inhibition of Ang II-induced VSMCs proliferation by the $\mathrm{AT}_{2} \mathrm{R}$ inhibitor PD123319 in SHR VSMCs. However, the inhibition of Ang II-induced VSMCs proliferation by PD123319 was abrogated in Sulf1 siRNA-transfected SHR VSMCs (Fig. 6B). Sulfs did not alter the inhibitory effects of losartan and PD123319 on Ang II-induced VSMCs proliferation in WKY VSMCs.

\section{DISCUSSION}

In the present study, we demonstrated that Sulfs, both Sulf1 and Sulf2, have no direct effect on the basal or Ang II-induced 12-LO and ET-1 expressions as well as on the cell proliferation in SHR VSMC; however, we showed that Sulf1 mediates the inhibitory action of $\mathrm{AT}_{2} \mathrm{R}$ blocker on Ang II-induced 12-LO and ET-1 expression and cell proliferation in SHR VSMCs.

Study on Sulfs first began around 2001 with the cloning of murine and human Sulfs; the first demonstration of glucosamine 6-O-endosulfatases [4]. Thereafter, Sulfs have been studied mainly as positive or negative regulators of carcinogenesis, as well as important modulators of cell signaling.

In has previously been determined that migration and proliferation of VSMCs induce intimal thickening as an important feature of hypertensive vasculature. The maintenance
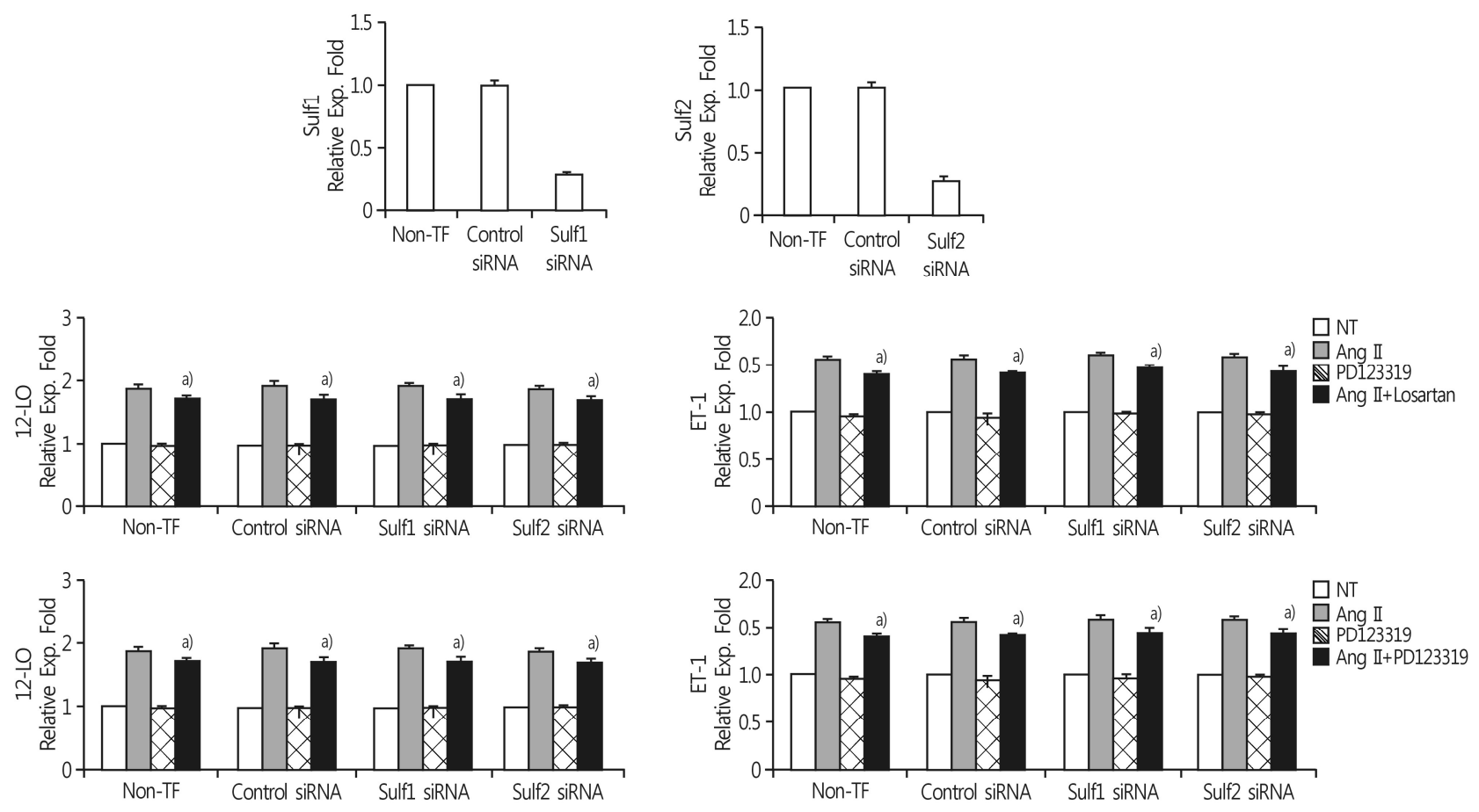

Fig. 4. Both of Sulf1 and Sulf2 do not mediate inhibition of Ang II-induced 12-LO and ET-1 expression by AT 2 R inhibitor in WKY VSMCs. WKY VSMCs were plated on 6-well plates, grown to $90 \%$ confluence, and transfected with Sulf1 or control siRNA oligomers $(50 \mathrm{nmol} / \mathrm{L})$. Total RNAs were analyzed by real-time PCR to confirm successful transfection. Additionally, WKY VSMCs were treated with or without Ang II $(0.1 \mu \mathrm{mol} / \mathrm{L})$ in the presence or absence of losartan $\left(\mathrm{AT}_{1} \mathrm{R}\right.$ inhibitor, $\left.10 \mu \mathrm{mol} / \mathrm{L}\right)$ or PD123319 (AT 2 R inhibitor, $10 \mu \mathrm{mol} / \mathrm{L}$ ) for $2 \mathrm{~h}$, after which total RNAs were analyzed by real-time PCR. Bars represent the means \pm SEM of three independent experiments. ${ }^{\text {a) }} p<0.05$ vs. non-transfected or transfected WKY VSMCs treated with Ang II. Sulf1, sulfatase 1; Ang II, angiotensin II; 12-LO, 12-lipoxigenase; ET-1, endothelin-1; $\mathrm{AT}_{1} \mathrm{R}$, angiotensin II type 1 receptor; $\mathrm{AT}_{2} \mathrm{R}$, angiotensin II type 2 receptor; WKY, Wistar-Kyoto rats; VSMCs, vascular smooth muscle cells. 
of normal 6-O sulfation levels by Sulf1 has been established to be important for the functions of VSMCs [15]. Overexpression or knockdown of the Sulf1 gene in normal VSMCs inhibits adhesion, while inducing proliferation and apoptosis. In osteoarthritis, expression of Sulfs is higher in osteoarthritic cartilage and old cartilage as compared with normal cartilage. This up-regulation of Sulfs mRNA expression and activity may alter the growth factor signaling pathways, leading to abnormal chondrocyte activation and cartilage degradation in osteoarthritis $[19,20]$. Therefore, it is possible that Sulfs play a functional role in hypertensive VSMCs, elevating the Sulf activity. However, to date, no study has evaluated the linkage between Sulfs and hypertensive VSMCs. The goal of this study was to evaluate the effects of Sulfs on hypertensive mediators and VSMCs proliferation in SHR VSMCs. We performed siRNA down-regulation to examine the role of Sulf1 or Sulf 2 on the expression of basal or Ang II-induced hypertensive mediators and VSMCs proliferation in SHR VSMCs.

As shown in our results, basal expression of Sulfs mRNAs and activity in SHR VSMCs were higher than those in normotensive WKY VSMCs. However, Ang II inhibited Sulfs mRNA expression and activity in SHR VSMCs. Therefore, we hypothesize that a high expression of Sulfs in SHR VSMCs may protect against hypertensive environment. On the other hand, Sulfs exerted no significant effects on the basal or Ang II-induced expression of hypertensive mediators, 12-LO and ET-1, in SHR VSMCs. However, unexpectedly, the inhibition of Ang II-induced 12-LO and ET-1 expressions by $\mathrm{AT}_{2} \mathrm{R}$ inhibitor, PD123319, was almost abrogated in Sulf1 siRNAtransfected SHR VSMCs. This suggests that although Sulfs may not directly affect the expression of Ang II-induced hypertensive mediators, Sulf1 may likely affect the $\mathrm{AT}_{2} \mathrm{R}$ pathway in the expression of Ang II-induced hypertensive mediators in SHR VSMCs. This result was not detected in Sulf1 siRNA-transfected WKY VSMCs (Fig. 4). Additionally, we observed the same results with other $\mathrm{AT}_{2} \mathrm{R}$ inhibitor, PD123177 (data not shown). Hypertensive environments in SHR VSMCs are different from normal environments in WKY VSMCs. First, the expression of Sulfs in WKY VSMCs has been shown to be lower than that in SHR VSMCs, and productions of Ang II-induced signaling molecules, including vasoactive mediators, have been established to be elevated in SHR relative
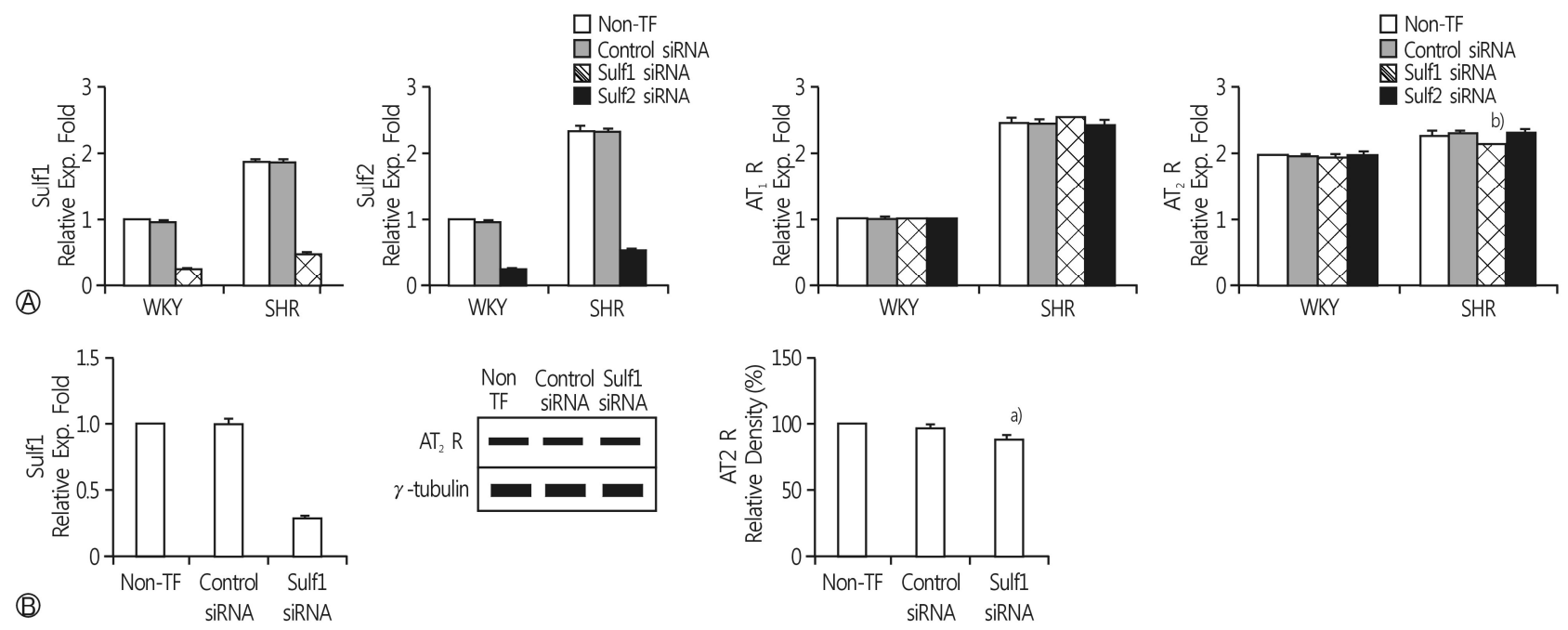

Fig. 5. Sulf1 affects expression of $\mathrm{AT}_{2} \mathrm{R}$ mRNA in SHR VSMCs. (A) WKY and SHR VSMCs were plated on 6-well plates, grown to a confluency of $90 \%$, and then transfected with Sulf1, Sulf2, or control siRNA oligomers (50 nmol/L). Total RNAs from transfected or non-transfected VSMCs were analyzed by real-time PCR to confirm successful transfection, after which $\mathrm{AT}_{1} \mathrm{R}$ and $\mathrm{AT}_{2} \mathrm{R}$ mRNA levels were measured. Bars represent means \pm SEM from three independent experiments. Non-TF: non-transfected WKY or SHR VSMCs. ${ }^{\text {b) }} p<0.01$ vs. control siRNA-transfected SHR VSMCs, (B) SHR VSMCs were transfected with Sulf1 or control siRNA oligomers $(50 \mathrm{nmol} / \mathrm{L}) \cdot \mathrm{AT}_{2} \mathrm{R}$ protein expression was determined by immunoblotting and densitometric analyses. Non-TF: non-transfected SHR VSMCs. Data shown are representative of three independent experiments. Bars represent the means \pm SEM of three independent experiments. ${ }^{\text {a) }} p<0.05$ vs. control siRNA-transfected SHR VSMCs. Sulf1, sulfatase 1; Sulf2, sulfatase 2; AT 1 R, angiotensin II type 1 receptor; $\mathrm{AT}_{2} \mathrm{R}$, angiotensin II type 2 receptor; WKY, Wistar-Kyoto rats; SHR, spontaneously hypertensive rats; VSMCs, vascular smooth muscle cells. 
to normotensive WKY [21-23]. Therefore, various environments between SHR and WKY VSMCs may give rise to differ- ent response to Sulfs.

Ang II a well-known vasoconstrictor and blood pressure
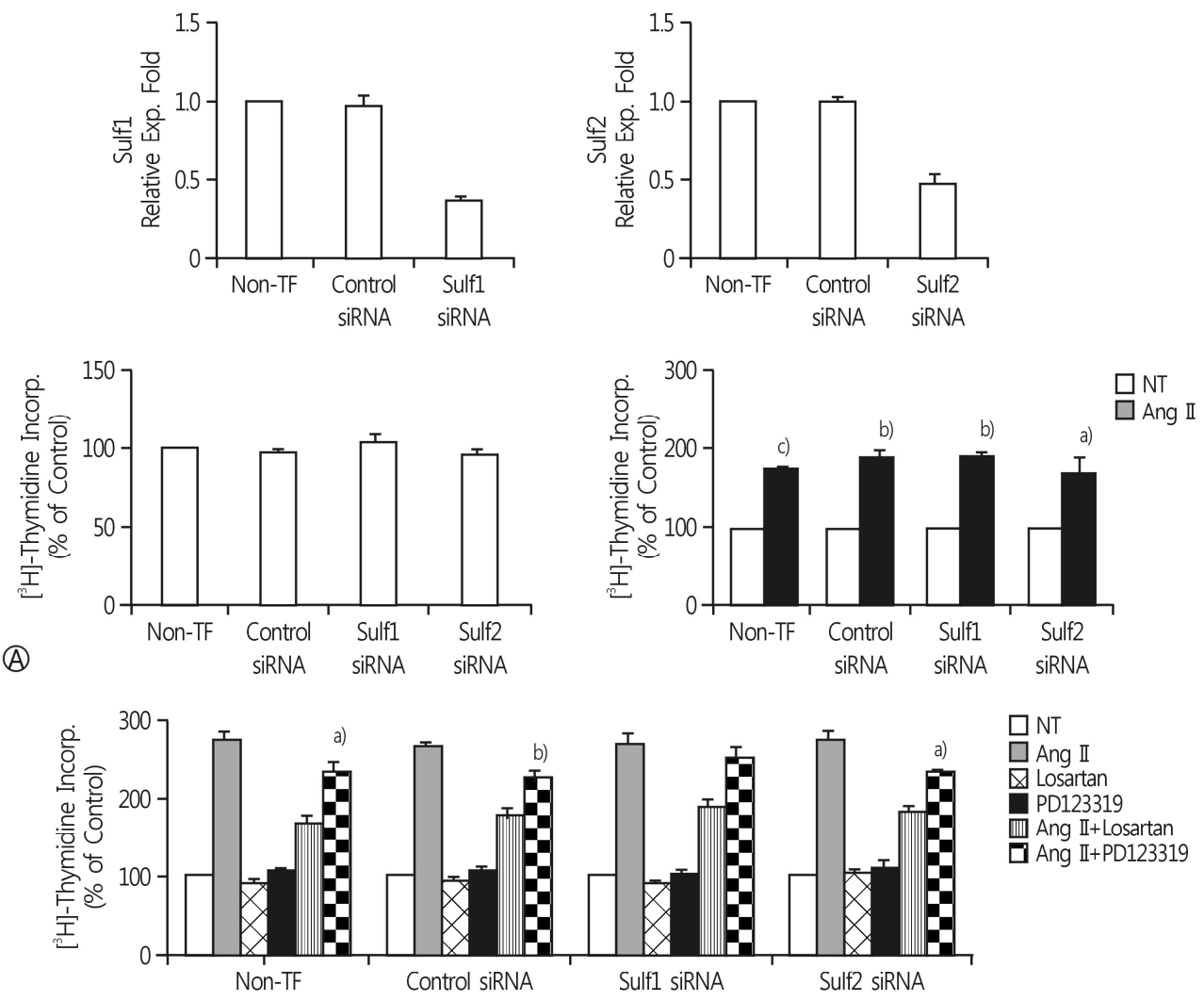

口NT

$\square$ Ang II

Q Losartan

血 Ang I+Losartan

D Ang $\mathbb{I}+$ PD123319

SHR VSMCS

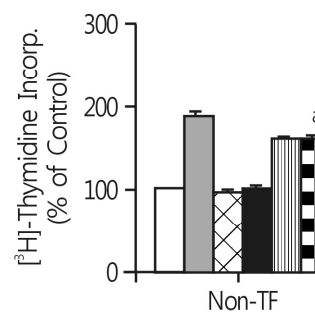

(B)

Fig. 6. Inhibition of Ang II-induced VSMCs proliferation by $\mathrm{AT}_{2} \mathrm{R}$ inhibitor, PD123319, is mediated through Sulf1 in SHR VSMCs. (A) SHR VSMCs were plated on 24-well plates, grown to $90 \%$ confluence, and then transfected with Sulf1, Sulf2, or control siRNA oligomers $(50 \mathrm{nmol} / \mathrm{L})$. Total RNAs were analyzed by real-time PCR to confirm successful transfection, after which SHR VSMCs were then untreated (NT) or treated with Ang II $(0.1 \mu \mathrm{mol} / \mathrm{L})$ for $24 \mathrm{~h}$ in medium containing $\left[{ }^{3} \mathrm{H}\right]$-thymidine $(1 \mu \mathrm{Ci} / \mathrm{mL})$. $\left[{ }^{3} \mathrm{H}\right]$-thymidine incorporation is shown on the Y-axis. Bars represent means \pm SEM from four independent experiments. ${ }^{\text {a) }} p<0.05,{ }^{\text {b) }} p<0.01,{ }^{\text {c) }} p<0.001$ vs. non-transfected or transfected untreated SHR VSMCs. (B) Non-transfected or transfected WKY or SHR VSMCs VSMCs were treated with or without Ang II (0.1 $\mu \mathrm{mol} / \mathrm{L}$ ) in the presence or absence of losartan (AT 1 R antagonist, $10 \mu \mathrm{mol} / \mathrm{L}$ ) or PD123319 (AT 2 R antagonist, $10 \mu \mathrm{mol} / \mathrm{L})$ for $24 \mathrm{~h}$ in medium containing $\left[{ }^{3} \mathrm{H}\right]$-thymidine $(1 \mu \mathrm{Ci} / \mathrm{mL})$. Bars represent the means \pm SEM of four independent experiments. ${ }^{\text {a) }} p<0.05,{ }^{b} p<0.01$ vs. non-transfected or transfected WKY or SHR VSMCs treated with Ang II. Ang II, angiotensin II; $\mathrm{AT}_{1} \mathrm{R}$, angiotensin II type 1 receptor; $\mathrm{AT}_{2} \mathrm{R}$, angiotensin II type 2 receptor; Sulf1, sulfatase 1; Sulf2, sulfatase 2; siRNA, small interfering RNA; WKY, WistarKyoto rats; SHR, spontaneously hypertensive rats; VSMCs, vascular smooth muscle cells. 
regulator, and the regulatory role of Ang II in blood pressure is mediated via two receptors, $\mathrm{AT}_{1} \mathrm{R}$ and $\mathrm{AT}_{2} \mathrm{R}[24,25]$. The expression of $\mathrm{AT}_{1} \mathrm{R}$ is generally higher than that of $\mathrm{AT}_{2} \mathrm{R}$ in VSMCs [24]. The major actions of Ang II, including vasoconstriction, inflammation, and VSMCs proliferation, are mediated through the $\mathrm{AT}_{1} \mathrm{R}$ pathway [25]. In contrast, $\mathrm{AT}_{2}$ $\mathrm{R}$ antagonizes the up-regulatory actions of $\mathrm{AT}_{1} \mathrm{R}$ in the hypertensive vasculature [24]. The expression levels of $\mathrm{AT}_{1} \mathrm{R}$ and $\mathrm{AT}_{2} \mathrm{R}$, including hypertensive mediators, in SHR VSMCs have been shown to be significantly higher than those in normotensive WKY VSMCs [18]. In our previous studies, the down-regulatory effect of CCL5 on the expression of Ang IIinduced hypertensive mediators has been shown to be mediated through the $\mathrm{AT}_{2} \mathrm{R}$ pathway [26]. In addition, the expression of IL-10-induced dimethylarginine dimethylaminohydrolase, a down-regulator of hypertension, has been mediated by the $\mathrm{AT}_{2} \mathrm{R}$ pathway [27]. Namely, an association between $\mathrm{AT}_{2} \mathrm{R}$ pathway and anti-hypertensive actions of IL-10 and CCL5 in SHR VSMCs has been determined [26-28]. However, the functional roles of $\mathrm{AT}_{2} \mathrm{R}$ remain controversial. Until now, the relationship between Ang II receptors and Sulfs has not been evaluated in hypertension studies. In this study, Sulfs did not affect $\mathrm{AT}_{1} \mathrm{R}$ expression in SHR VSMCs, whereas Sulf1 was associated with the expression of $\mathrm{AT}_{2} \mathrm{R}$ in SHR VSMCs. Although the rate of reduction of $\mathrm{AT}_{2} \mathrm{R}$ expression was low in Sulf1 siRNA-transfected SHR VSMCs, a reduction of $\mathrm{AT}_{2} \mathrm{R}$ expression mediated by down-regulation of Sulf1 was statistically significant. Moreover, the inhibitory effect of $\mathrm{AT}_{2} \mathrm{R}$ inhibitor on expression of Ang II-induced hypertensive mediators was abrogated in Sulf1 siRNAtransfected SHR VSMCs, similar to that in $\mathrm{AT}_{2} \mathrm{R}$ siRNAtransfected SHR VSMCs (data not shown). Ang II-induced VSMCs proliferation was also mediated in both pathways. However, the inhibitory effect of $\mathrm{AT}_{2} \mathrm{R}$ inhibitor on Ang II-induced VSMCs proliferation was abrogated in Sulf1 siRNA-transfected SHR VSMCs and Ang II-induced hypertensive mediators. Taken together, these results suggest that the maintenance of $\mathrm{AT}_{2} \mathrm{R}$ expression via Sulf1 on the $\mathrm{AT}_{2}$ $\mathrm{R}$ pathway plays an important role in the inhibition of $\mathrm{AT}_{2}$ $\mathrm{R}$ inhibitor on Ang II-induced hypertensive mediator expression and cell proliferation in SHR VSMCs.

In general, Sulf1 has been known to inhibit cancer cell proliferation [29,30]. Moreover, Sulf1 expression has been established to be down-regulated in ovarian, breast, and hep- atocellular cancers $[10,11,29]$. Dysregulated Sulf1 activity has also been shown to be associated with increased VSMCs proliferation [15]. Contrastingly, Sulf2 has been known to increase tumor cell proliferation [2,31]. Abnormal VSMCs proliferation is a characteristic feature of vascular hypertension, contributing to the harmful remodeling of blood vessels. Therefore, we observed the effect of Sulfs on hypertensive VSMCs proliferation. Unexpectedly, Sulf1 and Sulf2 had no effect on the basal or Ang II-induced proliferation of VSMCs in SHR. Since Sulfs are extracellular proteins, they could affect VSMCs. Sala-Newby et al. [15] has reported that both overexpression and down-regulation of Sulf1 can induce VSMCs proliferation. Although mRNA expression and activity of Sulfs were elevated in SHR VSMCs, neither Sulf1 nor Sulf2 had any effect on basal or Ang II-induced proliferation of VSMCs in SHR. Thus, the mechanism of Sulf1 in the context of VSMCs proliferation between hypertensive and normotensive VSMCs must further be elucidated.

In contrast to Sulf1, Sulf2 showed to have no effect on the inhibition of $\mathrm{AT}_{2} \mathrm{R}$ blockade regarding Ang II-induced hypertensive mediator expression and proliferation in SHR VSMCs. Moreover, Sulf2 did not affect $\mathrm{AT}_{2} \mathrm{R}$ expression in SHR VSMCs. Although the structures of Sulf1 and Sulf2 are similar with a highly conserved $\mathrm{N}$-terminal region domains they show different structures in the $\mathrm{COOH}$-terminal regions. The domains of their $\mathrm{COOH}$-terminal regions bind to the heparin sulfate chains. Therefore, differences in the heparin sulfate binding sites of the $\mathrm{COOH}$-terminal domains of Sulf1 and Sulf2 may result in functional differences. Whereas Sulf1 has a tumor suppressive effect via the inhibition of receptor tyrosine kinase signaling by desulfation of HSPG, Sulf 2 has an oncogenic effect via the activation of receptor tyrosine kinases [31,32].

In conclusion, Sulf1 mediates the inhibitory effect of $\mathrm{AT}_{2}$ $\mathrm{R}$ inhibitor on Ang II-induced hypertensive mediator expression and proliferation in SHR VSMCs. Namely, Sulf1 is associated with the $\mathrm{AT}_{2} \mathrm{R}$ pathway via the maintenance of $\mathrm{AT}_{2}$ $\mathrm{R}$ expression or an unknown action mechanism in SHR VSMCs. Although further study is required to better elucidate the discrete mechanisms of Sulf1 in the $\mathrm{AT}_{2} \mathrm{R}$ pathway in SHR VSMCs, this study demonstrates a new role of Sulf1 on $\mathrm{AT}_{2} \mathrm{R}$ pathway in Ang II-induced hypertensive effects in SHR VSMCs. 


\section{ACKNOWLEDGEMENT}

This research was supported by the Basic Science Research Program through the National Research Foundation of Korea (NRF) funded by the Ministry of Education (2015R1D1A1A 01057212).

\section{CONFLICT OF INTEREST}

The authors declare no conflict of interest.

\section{ORCID}

Hee Sun Kim, https://orcid.org/0000-0002-2000-4071

Hye Young Kim, https://orcid.org/0000-0001-7344-1526

\section{REFERENCES}

1. Buono M, Cosma MP. Sulfatase activities towards the regulation of cell metabolism and signaling in mammals. Cell Mol Life Sci 2010;67:769-80.

2. Sardiello M, Annunziata I, Roma G, Ballabio A. Sulfatases and sulfatase modifying factors: an exclusive and promiscuous relationship. Hum Mol Genet 2005;14:3203-17.

3. Nagamine S, Koike S, Keino-Masu K, Masu M. Expression of a heparan sulfate remodeling enzyme, heparan sulfate 6-Oendosulfatase sulfatase FP2, in the rat nervous system. Brain Res Dev Brain Res 2005;159:135-43.

4. Morimoto-Tomita M, Uchimura K, Werb Z, Hemmerich S, Rosen SD. Cloning and characterization of two extracellular heparin-degrading endosulfatases in mice and humans. J Biol Chem 2002;277(51):49175-85.

5. Honke K, Taniguchi N. Sulfotransferases and sulfated oligosaccharides. Med Res Rev 2002;22:637-54.

6. Langsdorf A, Do AT, Kusche-Gullberg M, Emerson CP Jr, Ai X. Sulfs are regulators of growth factor signaling for satellite cell differentiation and muscle regeneration. Dev Biol 2007;311:464-77.

7. Dai Y, Yang Y, MacLeod V, Yue X, Rapraeger AC, Shriver Z, et al. HSulf-1 and HSulf-2 are potent inhibitors of myeloma tumor growth in vivo. J Biol Chem 2005;280(48):40066-73.

8. Holst CR, Bou-Reslan H, Gore BB, Wong K, Grant D, Chalasani S, et al. Secreted sulfatases Sulf1 and Sulf2 have overlapping yet essential roles in mouse neonatal survival. PLoS One 2007;2:e575.

9. Ratzka A, Kalus I, Moser M, Dierks T, Mundlos S, Vortkamp A. Redundant function of the heparan sulfate 6-O-endosulfatases Sulf1 and Sulf2 during skeletal development. Dev Dyn 2008;237:339-53.
10. Narita K, Staub J, Chien J, Meyer K, Bauer M, Friedl A, et al. HSulf-1 inhibits angiogenesis and tumorigenesis in vivo. Cancer Res 2006;66:6025-32.

11. Zhang H, Newman DR, Sannes PL. HSULF-1 inhibits ERK and AKT signaling and decreases cell viability in vitro in human lung epithelial cells. Respir Res 2012;13:69.

12. Morimoto-Tomita M, Uchimura K, Bistrup A, Lum DH, Egeblad M, Boudreau N, et al. Sulf-2, a proangiogenic heparan sulfate endosulfatase, is upregulated in breast cancer. Neoplasia 2005;7:1001-10.

13. Uchimura K, Morimoto-Tomita M, Bistrup A, Li J, Lyon M, Gallagher J, et al. HSulf-2, an extracellular endoglucosamine6-sulfatase, selectively mobilizes heparin-bound growth factors and chemokines: effects on VEGF, FGF-1, and SDF-1. BMC Biochem 2006;7:2.

14. Heintz B, Stöcker G, Mrowka C, Rentz U, Melzer H, Stickeler E, et al. Decreased glomerular basement membrane heparan sulfate proteoglycan in essential hypertension. Hypertension 1995;25:399-407.

15. Sala-Newby GB, George SJ, Bond M, Dhoot GK, Newby AC. Regulation of vascular smooth muscle cell proliferation, migration and death by heparan sulfate 6-O-endosulfatase1. FEBS Lett 2005;579:6493-8.

16. Kim HY, Kang YJ, Song IH, Choi HC, Kim HS. Upregulation of interleukin-8/CXCL8 in vascular smooth muscle cells from spontaneously hypertensive rats. Hypertens Res 2008;31: 515-23.

17. Kim JH, Kim HS. Downregulation of angiotensin II-induced 12-lipoxygenase expression and cell proliferation in vascular smooth muscle cells from spontaneously hypertensive rats by CCL5. Korean J Physiol Pharmacol 2009;13:385-92.

18. Kim HY, Choi JH, Kang YJ, Park SY, Choi HC, Kim HS. Reparixin, an inhibitor of CXCR1 and CXCR2 receptor activation, attenuates blood pressure and hypertension-related mediators expression in spontaneously hypertensive rats. Biol Pharm Bull 2011;34:120-7.

19. Otsuki S, Taniguchi N, Grogan SP, D'Lima D, Kinoshita M, Lotz M. Expression of novel extracellular sulfatases Sulf-1 and Sulf-2 in normal and osteoarthritic articular cartilage. Arthritis Res Ther 2008;10:R61.

20. Tsai TT, Ho NY, Fang HC, Lai PL, Niu CC, Chen LH, et al. Increased sulfatase 1 gene expression in degenerative intervertebral disc cells. J Orthop Res 2015;33:312-7.

21. Sasaki M, Hori MT, Hino T, Golub MS, Tuck ML. Elevated 12-lipoxygenase activity in the spontaneously hypertensive rat. Am J Hypertens 1997;10:371-8.

22. Wu R, Millette E, Wu L, de Champlain J. Enhanced superoxide anion formation in vascular tissues from spontaneously hypertensive and desoxycorticosterone acetate-salt hypertensive rats. J Hypertens 2001;19:741-8.

23. Kim HY, Jeong DW, Park HS, Lee TY, Kim HS. Comparison of 12-lipoxygenase expression in vascular smooth muscle cells from old normotensive Wistar-Kyoto rats with spontaneously hypertensive rats. Hypertens Res 2013;36:65-73.

24. Horiuchi M, Lehtonen JY, Daviet L. Signaling mechanism of the AT2 angiotensin II receptor: crosstalk between AT1 
and AT2 receptors in cell growth. Trends Endocrinol Metab 1999; 10:391-6.

25. Guo F, Chen XI, Wang F, Liang X, Sun YX, Wang YJ. Role of angiotensin II type 1 receptor in angiotensin II-induced cytokine production in macrophages. J Interferon Cytokine Res 2011;31:351-61.

26. Kim HY, Cha HJ, Kim HS. CCL5 upregulates activation of AMP-activated protein kinases in vascular smooth muscle cells of spontaneously hypertensive rats. Cytokine 2014;67:77-84.

27. Kim HY, Kim HS. Dimethylarginine dimethylaminohydrolase1 mediates inhibitory effect of interleukin-10 on angiotensin II-induced hypertensive effects in vascular smooth muscle cells of spontaneously hypertensive rats. Cytokine 2016;77: 203-10.

28. Kim HY, Kim JH, Kim HS. Effect of CCL5 on dimethylarginine dimethylaminohydrolase- 1 production in vascular smooth muscle cells from spontaneously hypertensive rats. Cytokine
2013;64:227-33.

29. Li J, Mo ML, Chen Z, Yang J, Li QS, Wang DJ, et al. HSulf-1 inhibits cell proliferation and invasion in human gastric cancer. Cancer Sci 2011;102:1815-21.

30. Liu CT, Zhu ST, Li P, Wang YJ, Zhang H, Zhang ST. SULF1 inhibits proliferation and invasion of esophageal squamous cell carcinoma cells by decreasing heparin-binding growth factor signaling. Dig Dis Sci 2013;58:1256-63.

31. Yang JD, Sun Z, Hu C, Lai J, Dove R, Nakamura I, et al. Sulfatase 1 and sulfatase 2 in hepatocellular carcinoma: associated signaling pathways, tumor phenotypes, and survival. Genes Chromosomes Cancer 2011;50:122-35.

32. Lai JP, Sandhu DS, Yu C, Han T, Moser CD, Jackson KK, et al. Sulfatase 2 up-regulates glypican 3, promotes fibroblast growth factor signaling, and decreases survival in hepatocellular carcinoma. Hepatology 2008;47:1211-22. 